\title{
Low-Energy Tandem Mass Spectrometry of the Molecular Ion Derived from Fatty Acid Methyl Esters: A Novel Method for Analysis of Branched-Chain Fatty Acids
}

\author{
Joseph A. Zirrolli and Robert C. Murphy \\ National Jewish Center for Immunology and Respiratory Medicine, Denver, Colorado, USA
}

\begin{abstract}
Low-energy collision-induced dissociation (CID) of the molecular ions of fatty acid methyl esters obtained by electron ionization $(70 \mathrm{eV})$ decompose in the tandem quadrupole mass spectrometer to yield a regular homologous series of carbomethoxy ions. Even at energies up to $200 \mathrm{eV}\left(E_{\text {lab }}\right)$, primarily carbomethoxy ions are present, with the most abundant found at $m / z 101$ at higher energies. The lack of any other CID ions, including $m / z 74$ (McLafferty rearrangement) or $m / z 87$, suggest a rearranged molecular ion structure on leaving the first quadrupole mass analyzer. Analyses of various stable isotope variants support the hypothesis of alkyl radical migration to the carboxy carbonyl oxygen atom, with subsequent radical site directed cleavage either with or without a cyclization event. Decomposition of the molecular ions $(70 \mathrm{eV})$ of several methyl branched fatty acid methyl esters, including phytanic acid, iso-methyl and anteiso-methyl branched acids, and tuberculostearic acid, reveals enhanced radical site cleavage at the alkyl branching positions. This method can be used to readily determine methyl (or alkyl) branching positions in a saturated fatty acid methyl ester. ( $(\mathrm{Am}$ Soc Mass Spectrom 1993, 4, 223-229)
\end{abstract}

$\mathrm{F}$ Tatty acid methyl ester derivatives were the first lipid substances to be studied in detail by mass spectrometry. Ryhage and Stenhagen [1-4] in the late 1950 s and early 1960 s investigated the behavior of these compounds, and the results of these studies have served as a basis for much of our understanding of events taking place during electron ionization (EI) mass spectrometry. The EI mass spectra of saturated straight-chain fatty acid methyl esters are very similar, having a reasonably abundant molecular ion and a characteristic base peak at $m / z 74$, which is a product of the well-recognized McLafferty rearrangement process $[5,6]$. Other characteristic ions include an ion at $m / z$ 87, the first member of the carbomethoxy ion series $\left(\left[\left(\mathrm{CH}_{2}\right)_{n} \mathrm{CO}_{2} \mathrm{CH}_{3}\right]^{+}\right.$, where $n=1,2,3,4, \ldots$; $m / z 73,87,101,115, \ldots)$, a hydrocarbon series of ions that are abundant in the low-mass region, and an acylium ion [M - 31]. Dinh-Nguyen [7] used several isotope label analogs of these compounds to facilitate investigation of the detailed mechanisms taking place following EI to explain the various fragment ions observed. With the advent of tandem mass spectrometry, the opportunity to further investigate the mechanism of formation of abundant ions by alternative tech-

Address reprint requests to Robert C. Murphy, Department of Pedi atrics, National Jewish Center, 1400 Jackson Street, Denver, CO 80206. niques has become possible. Unlike the fragment ions observed in the ion source following EI, collision-induced dissociation (CID) of the molecular ion yielded only one series of ions corresponding in mass to the carbomethoxy series. One of the analytically useful features of this process was found to be in the facile structural characterization of branched-chain fatty acid methyl esters.

\section{Experimental}

Free fatty acids were purchased from Sigma Chemical Co. (St. Louis, MO), and methyl esters were prepared by reaction with an ethereal solution of diazomethane. Ethyl octadecanoate was obtained by reaction of the free acid with $3 \%$ anhydrous $\mathrm{HCl}$ in ethanol.

\section{Stable Isotope Variants}

Hexadecanoic acid- $1,2,3,4-{ }^{13} \mathrm{C}\left(99.3\right.$ atom $\left.\%{ }^{13} \mathrm{C}\right)$ and $\left[18,18,18-\mathrm{D}_{3}\right]$ octadecanoic acid (99.5 atom \% D) were obtained from MSD Isotopes (Montreal, Canada). $\mathrm{D}_{3}-$ methyl ester of octadecanoic acid was prepared by first treating the fatty acid with an ethereal solution of deuterium oxide $(99.8$ atom \% D; Aldrich Chemical Co., Milwaukce, WI) and then reacting with an ethereal solution of deuterated diazomethane. Methyl [2,2- 
$\mathrm{D}_{2}$ ]octadecanoate was prepared by exchanging the free acid in $70 \%$ methanol-OD $(99.5$ atom \% D; Aldrich Chemical $\mathrm{Co}$.) in deuterium oxide $(\mathrm{v} / \mathrm{v})$ and sodium methoxide $(5 \mathrm{M})$ overnight at room temperature with constant stirring. The sodium methoxide was prepared immediately before use from sodium and methanolOD. After the exchange was complete, the solution was acidified with formic acid; octadecanoic acid was extracted with hexane, the hexane solution evaporated to dryness under nitrogen, and the methyl ester formed with diazomethane.

\section{Branched-Chain Acids}

Methyl tuberculoslearate was ubtained following transesterification from mycobacterial glycolipid (a gift from Dr. Michael McNeil, Colorado State University, $\mathrm{Ft}$. Collins, $\mathrm{CO}$ ) with $5 \%$ anhydrous $\mathrm{HCl}$ in methanol for $4 \mathrm{~h}$ at $80^{\circ} \mathrm{C}$. The sample was then dried under nitrogen, washed once with methanol, dried again, and then dissolved in hexane. The same was analyzed by combined gas chromatography/tandem mass spectrometry (MS/MS) because it was a mixture of fatty acid esters.

Phytanic acid (3,7,11,15-tetramethyl-hexadecanoic acid) was prepared from phytol (Aldrich Chemical Co.) by initial hydrogenation with $5 \%$ palladium on carbon in ethanol under a hydrogen atmosphere. The acid was then formed by treatment with Jones' reagent (10 mg CrO $3,0.5 \mu \mathrm{L}$ of concentrated $\mathrm{H}_{2} \mathrm{SO}_{4}$ in $1 \mathrm{~mL}$ of acetone) for $5 \mathrm{~min}$ on ice, then at room temperature for an additional $15 \mathrm{~min}$. Water $(2 \mathrm{~mL})$ was then added, and the acid was extracted with ethyl acetate $(2 \times 3$ $\mathrm{mL}$ ).

\section{Mass Spectrometry}

Mass spectral analysis was performed with a TSQ 70 triple quadrupole mass spectrometer (Finnigan-MAT Corp., San Jose, CA) in either single or tandem scanning modes. Electron ionization was carried out at 70 $\mathrm{eV}$. Collisional activation was obtained in the second quadrupole region $(\mathrm{Q} 2)$ using argon (0.1-0.7 torr) and offset acceleration potentials ranging from 1 to $200 \mathrm{eV}$ laboratory frame of reference $\left(E_{\mathrm{lab}}\right)$. Product ion spectra were obtained by scanning the third quadrupole from $m / z 50$ to the molecular ion in $2 \mathrm{~s}$. Fatty acid esters (5-10 ng) were introduced into the mass spectrometer either with a direct insertion probe or via a gas chromatograph (Varian model 3400; Varian Associates, Walnut Creek, CA) with a $10-\mathrm{m} \times 0.25-\mathrm{mm}(0.25$ $\mu \mathrm{m}$ film thickness) DB-1 capillary column (J \& W Scientific, Folsom, CA) with helium as the carrier gas.

\section{Results}

\section{Saturated Fatty Acid Methyl Ester}

A comparison is shown in Figure 1 of the mass spectra obtained from methyl octadecanoate by EI (Figure 1a)
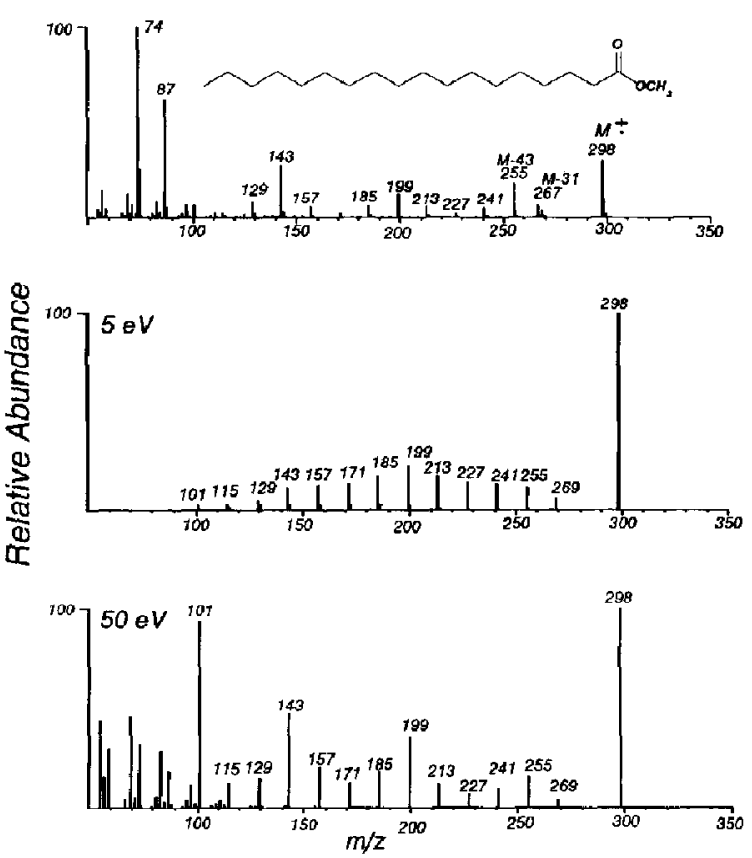

Figure 1. (a) El (70 eV) mass spectrum of methyl octadecanoate; (b) product ions following CID $\left(5 \mathrm{eV}, E_{1 \mathrm{ab}}\right)$ of the molecular ion ( $m / z$ 298) of methyl octadecanoate; and (c) product ions following CID $\left(50 \mathrm{eV}, E_{\mathrm{lab}}\right)$ of the molecular ion of methyl octadecanoate.

followed by collisional activation of the molecular ion (Figure 1b). The low-energy (5-eV $\mathrm{Q} 2$ offset voltage) activation yielded only ions of the carbomethoxy series $\left[\left(\mathrm{CH}_{2}\right)_{n} \mathrm{COOCH}_{3}\right]^{+}$. Remarkably, ions typical of the fatty acid methyl ester EI mass spectrum were completely absent. These included the McLafferty rearrangement ion $(m / z 74)$, all low-mass ions corresponding to the hydrocarbon series [both saturated $\left(\mathrm{C}_{n} \mathrm{H}_{2 n+1}\right)$ and unsaturated $\left.\left(\mathrm{C}_{n} \mathrm{H}_{2 n-1}\right)\right]$, and the acylium ion $(m / z 267)$ resulting from the loss of the methoxy radical. Rather, a singular and regular series of ions was produced that corresponded nominally to simple alkyl radical losses, beginning with ethyl ([M $29]^{+}$), that were identical in mass to the carbomethoxy series ions. The abundance of these ions was maximal, with a carbon length of $C_{10}-C_{12}(n=9-11$ in the carbomethoxy series) and decreased with either shorter or longer lengths of the carbomethoxy ion. This pattern was observed with fatty acid methyl esters of carbon lengths $C_{12}-C_{20}$. With longer chain fatty acid methyl esters, the series continued but with another abundance maximum occurring at approximately $\mathrm{C}_{17}-\mathrm{C}_{19}$ (for methyl tetracusanivate).

As the collisional energy was increased (Figure 1c), the pattern was changed, with the ion at $m / z \quad 101$ becoming the most abundant. The ion series $m / z 143$, 199,255 , etc., which was pronounced in the EI mass spectrum, was also enhanced, with higher collision energies as well as the formation of the hydrocatbon 
type ions; however, the McLafferty rearrangement ion was not observed under any collisional conditions (up to $200 \mathrm{eV}$ offset voltage and 1 torr pressure).

This series of carbomethoxy ions produced following collisional activation was observed only with straight-chain, saturated fatty acid methyl esters. The decomposition of unsaturated methyl esters yielded multiple series of ions from alkyl radical losses. The multiplicity of these series was dependent on the degree of unsaturation and probably indicates scrambling of the original double-bond positions. Collisional-induced dissociation of keto- and hydroxy-fatty acid methyl esters also failed to produce the regular pattern of carbomethoxy ions observed in Figure 1b; however, saturated, branched fatty acid methyl esters did yield similar ions under CID conditions, but the abundances of specific ions in the series were enhanced by the influence of the positions of branching, as is discussed in detail later.

\section{Stable Isotope-Labeled Fatty Acid Methyl Ester}

The influence of the ester functionality on the CID formation of carbomethoxy ions was studied using $D_{3}$-methyl and ethyl esters of octadecanoic acid. The molecular ion of $\mathrm{D}_{3}$-methyl octadecanoate was shifted by the expected $3 \mathrm{u}$ from $\mathrm{m} / z 298$ to 301 , as was the entire series of carbomethoxy ions formed by lowenergy $(5 \mathrm{eV})$ collisional activation. Likewise with higher collisional offset voltages $(>50 \mathrm{eV})$, the most abundant ion in the mass spectrum became $m / z$ 104, where $n=3$ in the carbomethoxy series. Again, a similar pattern was observed with low-energy collisional activation of ethyl octadecanoate, and all ions were shifted by the expected $14 \mathrm{u}$ from those obtained with the methyl ester; however, with higher energy collisional activation conditions, the most abundant product ion from CID of the molecular ion was observed at $m / z 87$ and not the expected $m / z 115$ for the $n=3$ ion of the series. This most likely resulted from further decomposition of this ion with loss of ethylene $(28 \mathrm{u})$ from the ester moiety. These results confirm inclusion of the ester moiety in the ion series formed by collisional activation and further support the formation of ions via alkyl radical losses.

More complex product ion spectra were obtained with specific labeling of the alkyl chain with either deuterium or carbon-13. As shown in Figure 2a, the low-energy CID spectra observed with methyl [2,2$\mathrm{D}_{2}$ ] octadecanoate shows extensive deuterium scrambling from their original positions. The carbomethoxy product ion series was now composed of triplets, with the most deuterium exchange occurring when $n=$ 7-11 and almost complete loss or retention of the label when the alkyl chain was short or long, respectively. With higher collision energy, the pattern of deuterium exchange remained the same; however, $m / z 101(n=$ 3) again became the most abundant product ion. Previously, the elegant work of Dinh-Nguyen [7] had docu-
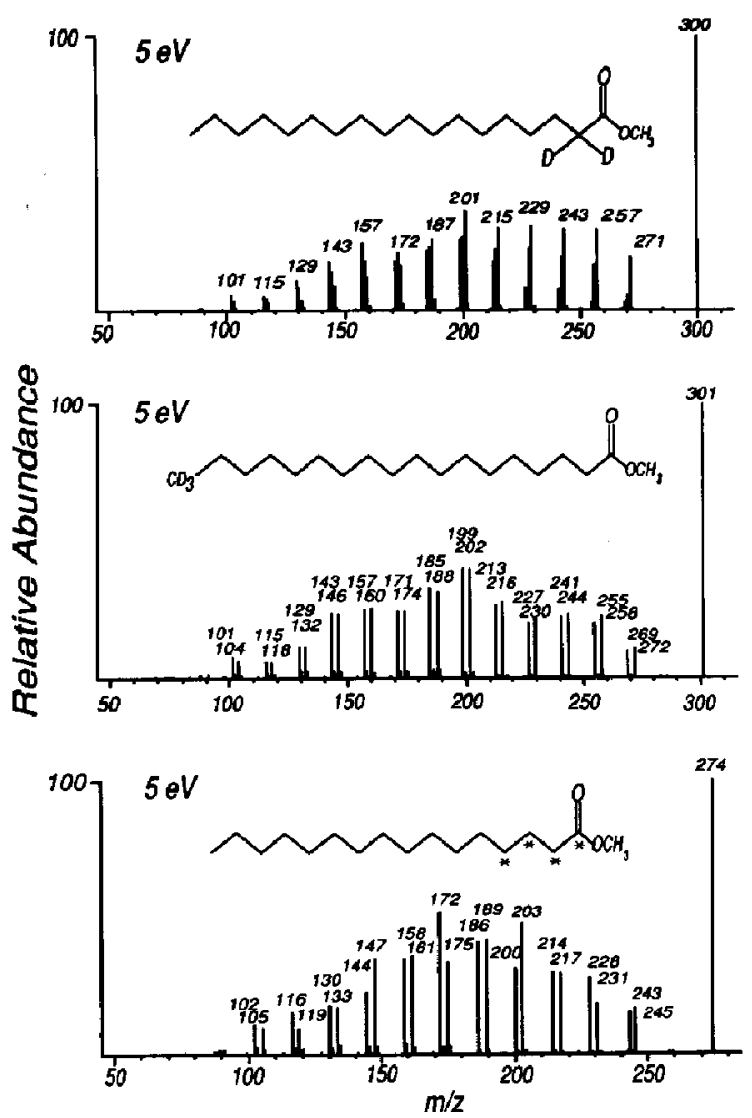

Figure 2. (a) Product ions following CID (5 eV, $E_{\mathrm{lab}}$ ) of the molecular ion ( $m / z 300)$ of methyl $\left[2,2-\mathrm{D}_{2}\right.$ loctadecanoate (EI, 70 $\mathrm{eV}$ ) (b) product ions following CID $\left(5 \mathrm{eV}, E_{\mathrm{lab}}\right)$ of the molecular ion $(m / z 301)$ of methyl $\left[18,18,18-\mathrm{D}_{3}\right]$ octadecanoate (EI, $70 \mathrm{eV}$ ); and (c) product ions following CID (5 eV, $E_{\text {lab }}$ ) of the molecular ion $(m / z 274)$ of methyl $\left[1,2,3,4-{ }^{13} \mathrm{C}_{4}\right]$ hexadecanoate (EI, $70 \mathrm{eV}$ ).

mented the extensive and complex deuterium exchange processes occurring in the ion source under EI conditions.

Source-initiated deuterium exchange reactions did not occur when the terminal methyl group was labeled [7]. This was also true of the collisional activation of molecular ions in the tandem quadrupole; however, the product ion spectra obtained from methyl $\left[18,18,18-\mathrm{D}_{3}\right]$ octadecanoate indicated a complex rearrangement of carbon atom constituents in the carbomethoxy ion series (Figure 2b). The product ion pattern now abserved was composed of doublets, each differing by $3 u$, implying two series of ions-one that had retained the terminal methyl group (with all three deuterium atoms) and one that lost this methyl group. The occurrence of only doublets always differing by $3 \mathrm{u}$ and no other ion multiplets indicated rearrangement of carbon constituents without any appreciable deuterium exchange. The range of this rearrangement process was demonstrated by the carbomethoxy ion in methyl $\left[18,18,18-\mathrm{D}_{3}\right]$ octadecanoate at $m / z 104(n=3)$, 
implying transfer of the terminal methyl group with its deuterium atoms (probably with $C_{16}-C_{17}$ as well) to $C_{1}$ and loss of the alkyl radical containing $C_{2}-C_{15}$. With low-energy collisional activation (Figure 2b), the equal abundance of each ion of the doublet suggests comparable rates of formation for both ions; however, with elevated collisional activation energies, the unlabeled carbomethoxy ion at $m / z 101$ predominated.

The complex rearrangement of carbon constituents was also observed with another stable isotope-labeled fatty acid methyl ester. As shown in Figure $2 c$, the low-energy CID of the molecular ion $(m / z 274)$ from methyl $\left[1,2,3,4-{ }^{13} \mathrm{C}_{4}\right]$ hexadecanoate produced another series of doublets of carbomethoxy ions, each differing by $3 \mathrm{u}$, except for the $[\mathrm{M}-29]^{+}$doublet at $\mathrm{m} / z 243$ and 245-a difference of $2 \mathrm{u}$ due to either loss of doubly carbon-13-labeled or unlabeled ethyl radical, respectively; however, all product ions retained at least one carbon-13, which was the $C_{1}$ carboxy carbon atom and which again supported a carbomethoxy ion structure. Rearrangement of alkyl constituents was comparable from the carboxy end of the carbon chain as well as from the methyl terminus because each ion of a doublet was similar in relative abundance. With higher collision energies, the most abundant product ion was observed for $n=3$ at $m / z 102$, reflecting loss of $C_{2}-C_{4}$ but retention of $C_{1}$. This is in contrast with the $n=3$ carbomethoxy ion $(m / z$ 101) from methyl $[18,18,18-$ $\mathrm{D}_{3}$ loctadecanoate (Figure $2 \mathrm{~b}$ ), where the terminal deuterium labels were also lost.

\section{Branched, Saturated Fatty Acid Methyl Ester}

Collisional activation of branched, saturated fatty acid methyl esters also yielded a regular series of carbomethoxy ions. With these compounds, a significant increase in the relative abundance of ions derived from specific radical losses on either side of the branching carbon was observed. This provided a method for the determination of alkyl branches in fatty acid methyl esters.

Positive detection of tuberculostearic acid (10methyl-octadecanoic acid) can be diagnostic of the presence of mycobacteria [8]. Although the EI mass spectrum of methyl tuberculostearate differs slightly from that of the saturated, straight-chain methyl ester (compare Figure 3a with Figure 1a), it is not possible to conclusively identify the methyl branch point at $\mathrm{C}_{10}$; however, the low-energy CID spectrum of methyl tuberculostearate (Figure $3 \mathrm{~b}$ ) is much different than that obtained from methyl octadecanoate (Figure 1b). With the branched analog, the regular pattern of the carbomethoxy ions was disrupted, and the most abundant product ions were observed at $m / z 171$ and 199, corresponding to $C_{9}-C_{10}$ and $C_{10}-C_{11}$ bond scission, respectively. Although the increased abundance of these ions indicated the branching positions, the mass difference of $28 \mathrm{u}$ between the ions indicated that the alkyl branch was a methyl group.

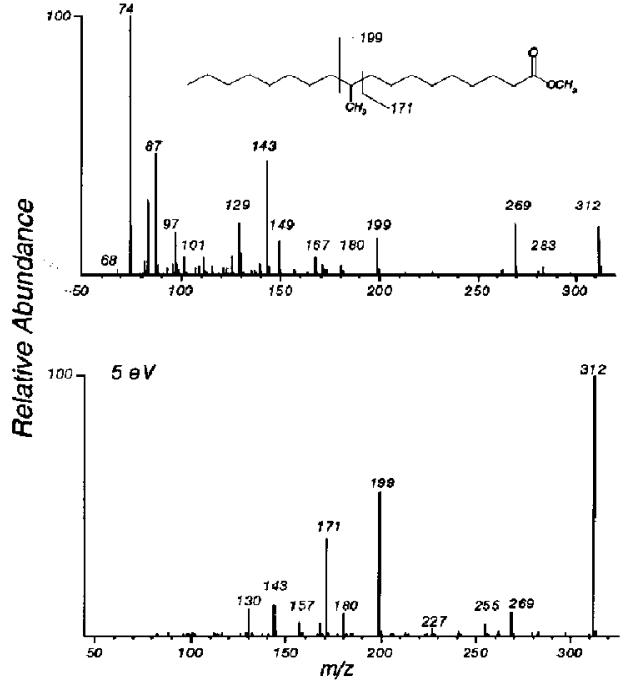

Figure 3. (a) EI $(70 \mathrm{eV})$ mass spectrum of the methyl ester of tuberculostearic acid (methyl 10-methyl-octadecanoate); (b) CID $\left(5 \mathrm{eV}, E_{\mathrm{lab}}\right)$ of the molecular ion $(\mathrm{m} / z \mathrm{312})$ of methyl 10-methyloctadecanoate.

Differentiation of iso-methyl and anteiso-methyl isomers of fatty acids is difficult solely on the basis of EI mass spectrometry $[6,9,10]$. As previously reported, there are few diagnostic ions to identify either the presence or the position of the methyl branches in the EI mass spectrum of methyl 13-methyl-pentadecanoate (Figure 4a) and methyl 14-methyl-pentadecanoate (Figure 5a). This information is readily obtained from the low-energy CID of their respective molecular ions. Product ions of relatively higher abundance at $\mathrm{m} / z 213$ and 241 clearly indicated the anteiso position of the methyl group in methyl 13-methyl-pentadecanoate (Figure 4b). At higher collisional energies (>50 eV), $\mathrm{m} / z 101$ becomes the most abundant product ion for methyl 13-methyl-pentadecanoate (data not shown).

In contrast, the CID spectrum of methyl 14-methylpentadecanoate showed the most abundant product ion at $m / z 227$ (Figure 5b), a shift of $14 \mathrm{u}$ less, indicating the iso position of the methyl group. This spectrum also shows a product ion corresponding to $\lceil\mathbf{M}-15\rceil^{+}$, although not strikingly abundant. This ion is not normally observed with straight-chain fatty acid methyl esters, but in this case its formation is facilitated by the branch group at $\mathrm{C}_{\mathbf{1 4}}$.

\section{Phytanic Acid}

Phytanic acid (3,7,11,15-tetramethylhexadecanoic acid) is related to phytol, an unsaturated alcohol constituent of chlorophyll. The base peak at $m / z 101$ in the EI mass spectrum of methyl phytanoate (Figure 6a) clearly showed this to be an a-methyl branched fatty acid; however, there were no ions that clearly indicated the positions of the remaining three methyl groups. Com- 


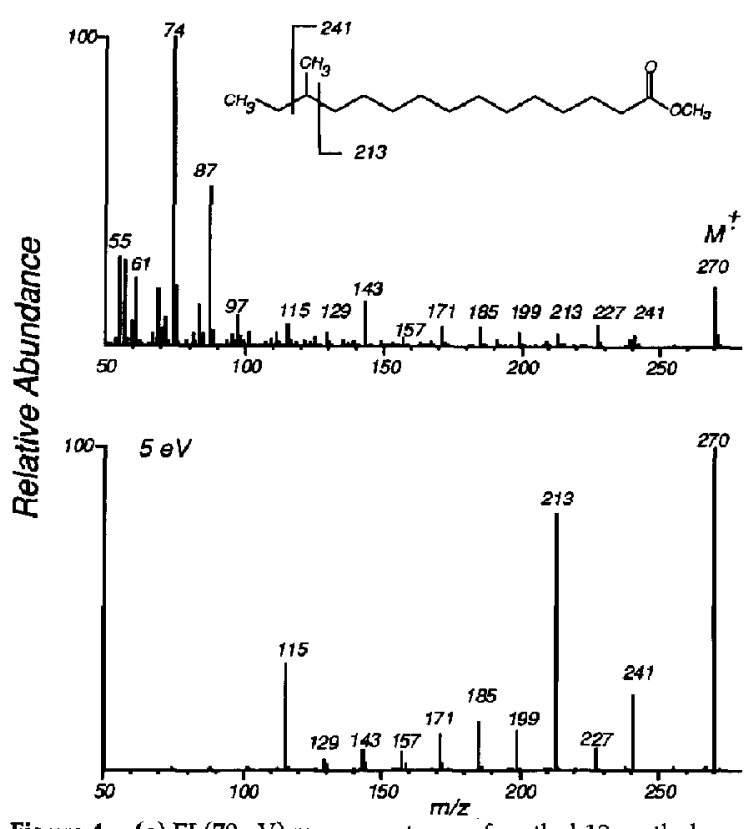

Figure 4. (a) EI (70 eV) mass spectrum of methyl 13-methylpentadecanoate; (b) CID (5 eV, $E_{\text {lab }}$ ) of the molecular ion $(m / z 270)$ of methyl 13-methylpentadecanoate.

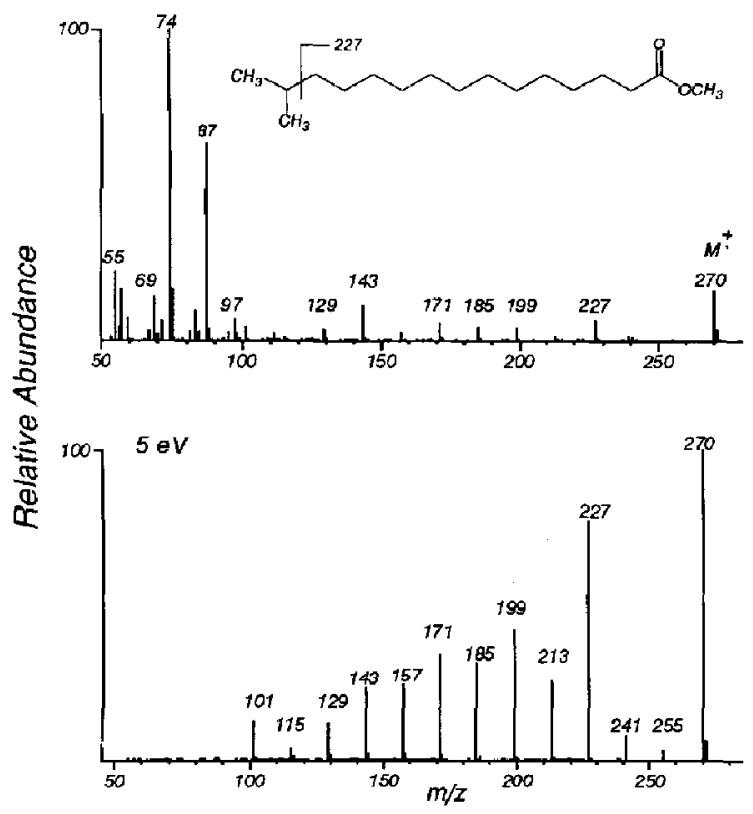

Figure 5. (a) EI (70 eV) mass spectrum of methyl 14-methylpentadecanoate; (b) CID (5 eV, $\left.E_{\text {lab }}\right)$ of the molecular ion ( $m / z 270$ ) of methyl 14-methylpentadecanoate.

plementary information was obtained from the low-energy CID mass spectrum (Figure 6b), which showed product ions from alkyl radical losses about the methyl branched carbons. The product ion pairs of $m / z 143$ and 171 and 213 and 241 marked the methyl groups at

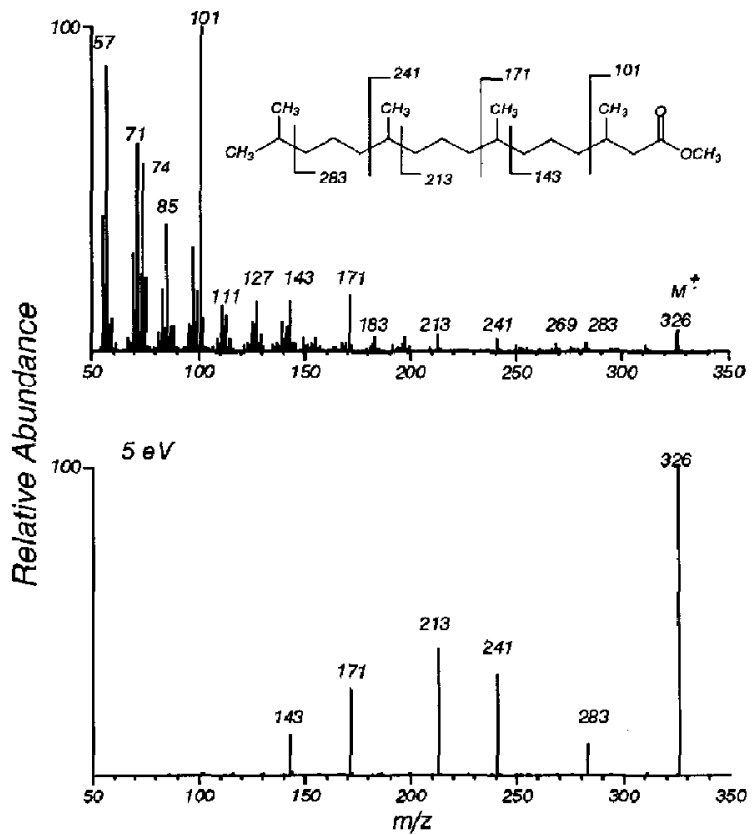

Figure 6. EI (70 eV) mass spectrum of methyl $3,7,11,15$-methylhexadecanoate (methyl phytanate); (b) $\mathrm{CID}\left(5 \mathrm{eV}, E_{\mathrm{lab}}\right.$ ) of the molecular ion ( $m / z 326)$ of methyl 3,7,11,15-tetradecanoate.

$C_{7}$ and $C_{11}$, respectively, whereas the ion at $m / z 283$ indicated the iso-methyl group. It is of interest to note that as the position of methyl group branching approaches the carbomethoxy moicty, abundance of the characteristic product ion decreases. With the methyl group at $\mathrm{C}_{3}$ for methyl phytanoate, almost no ion abundance is observed at $m / z 101$ or 73 . The absence of a product ion at $\mathrm{m} / \mathrm{z} 101$ (the base peak in the EI mass spectrum) was consistent with the carbon-chain rearrangements observed with methyl $[1,2,3,4-$ ${ }^{13} \mathrm{C}_{4}$ ] hexadecanoate and loss of the $\mathrm{C}_{2}-\mathrm{C}_{4}$ group containing the $C_{3}$ methyl substituent. This example clearly illustrates the complementary value of the EI mass spectrum and the low-energy CID of the molecular ion for characterization of branched-chain fatty acid methyl esters.

\section{Discussion}

The study of the EI and fragmentation of fatty acid methyl esters represents one of the earliest applications of mass spectrometry in the analysis of biomolecules as well as one of the most extensive [1-7]. These mass spectra, which are familiar to all organic mass spectroscopists, are characterized by the base peak at $m / z 74$ formed by the McLafferty rearrangement [5] and the periodic carbomethoxy ion series $\left[\left(\mathrm{CH}_{2}\right)_{n} \mathrm{COOCH}_{3}\right]^{+}$at $m / z 87,101,115,129$, etc. Indeed, the formation of the odd-electron ion $(\mathrm{m} / z \mathrm{z} 4)$ is probably the most common example of a $\gamma$-hydrogen rearrangement to an unsaturated group with subse- 
quent $\beta$-cleavage taught in basic courses on mass spectral interpretation. The absence of this ion during the collisional activation of the molecular ion from saturated fatty acid methyl esters was a most striking feature of these low-energy tandem mass spectra and posed fundamental questions about the structure(s) of the molecular ions leaving the ion source after formation at $70 \mathrm{eV}$ and the mechanism of subsequent activation and decomposition.

The other striking feature of these low-energy CID mass spectra was the sole formation of the carbomethoxy ion series. The formation of these ions with EI was originally studied by Dinh-Nguyen [7] in an exhaustive study involving gem-dideutero-, vicinal dideutero-, terminal $\mathrm{D}_{3}$-methyl, and the $\mathrm{D}_{3}$-methoxylabeled species of methyl octadecanoate. This work clearly showed the involvement of three distinct mechanisms in the formation of the carbomethoxy ions within the ion source. The first mechanism was the loss of a terminal alkyl radical, initiated by a hydrogen atom transfer to the carbonyl oxygen, leaving an alkyl radical that leads to adjacent carbon bond cleavage. $A$ second mechanism proposed by Budzikiewicz et al. [10] involved the complex scission of an internal methylene chain with a hydrogen transfer and rearrangement of the carbon skeleton, as shown in Scheme I for the $[\mathrm{M}-43]^{+}$ion. These two mechanisms might be expected to be competitive with the dominant process dependent on the specific ion formed. For example, the $18,18,18-\mathrm{D}_{3}$-methyl group was retained in the carbomethoxy series when $n>5$ but was not found at all when $n<6$. A third process [7] was an extensive but also position-specific hydrogen atom transfer among the skeleton carbon chain, as indicated by observed deuterium-hydrogen exchanges. This third process compounded both of the other two mechanisms. Thus, with methyl 2,2-dideutero-octadecanoate, formation of ions at $m / z 87(n=2)$ was shifted by $1 u ; m / z 255$ $(n=14)$ was also shifted by $1 u$; and $m / z 199(n=10)$ was shifted by 0,1 , or $2 \mathrm{u}$.

Similar processes were also clearly observed, but to a greater extent than in EI spectra, in the formation of carbomethoxy ions in the low-energy CID of $\mathrm{M}^{+\cdot}$ of straight-chain, saturated fatty acid methyl esters. As shown in Figure 2, two competitive processes result in the doublet series of carbomethoxy ions by losses of alkyl radicals. The low-energy CID mass spectrum of methyl $\left[2,2-\mathrm{D}_{2}\right]$ octadecanoate containing isotope multiplets of the carbomethoxy ion series (Figure 2) is also consistent with hydrogen-deuterium exchange occurring in both mechanisms, leading to alkyl radical losses.

The striking feature in the observed CID mass spectra was the lack of formation of ions at $m / z 74$ and 87 , the most abundant ions formed following EI. Collisional activation and decomposition of ions can drastically differ from source-formed ions based on the energy and time considerations of the processes involved. El source conditions favor highly energetic processes, especially at $70 \mathrm{eV}$. Collisional activation conditions in triple quadrupole mass spectrometers are relatively low in energy, whereas the opposite is the case in tandem-sector mass spectrometers [9, 11]; however, similar CID mass spectra as well as metastable ion spectra from the $\mathbf{M}^{+}$of fatty acid methyl esters were observed with tandem-sector mass spectrometers (personal communications, M. L. Gross and F. W. McLafferty, respectively). In these high-energy experiments, $m / z 74$ was not observed.

Considering the low energy used in the tandem quadrupole instrument and the fact that the same ions are observed as metastable ions suggest that the EI process imparts sufficient energy to induce the formation of these ions that leave the ion source as metastable ions. We do not believe that the decomposition mechanism is similar to that described as "charge-remote" mechanisms, which are observed typically at highenergy conditions. It is important to point out that the ions under investigation are radical cations, and we suggest that the radical site is driving the fragmentation process.

A further consideration is that the structure of "molecular ions"' leaving the first quadrupole region may be different than that initially formed in the EI source. If such molecular ions do not have a hydrogen transferred to or a radical site on the carbonyl oxygen atom (the initial steps in formation of $m / z 74$ and 87), then an entirely different decomposition pathway may be operative following $\mathrm{CA}$. To rationalize the observed CID mass spectra, we suggest here a rearranged molecular ion structure as it leaves the first quadrupole region (Scheme II). Activation and decomposition of this ion would not lead to formation of $m / z 74$ or 87 . Additionally, the loss of the methoxy radical to form the acylium ion $[\mathrm{M}-31]^{+}$, as observed in the EI mass

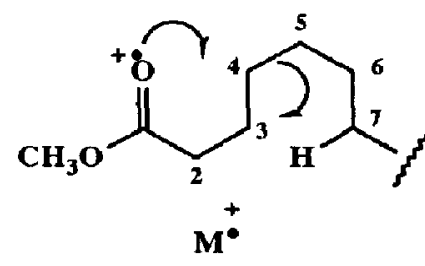

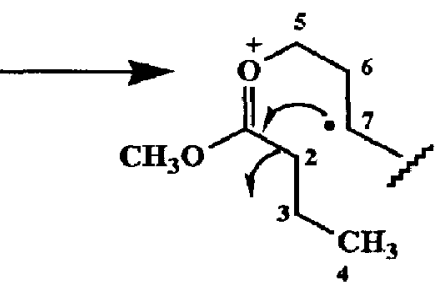

Scheme I

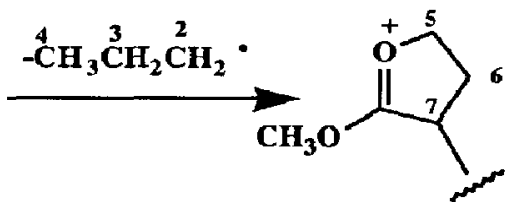

$\mathbf{M}-\mathbf{4 3}$ 


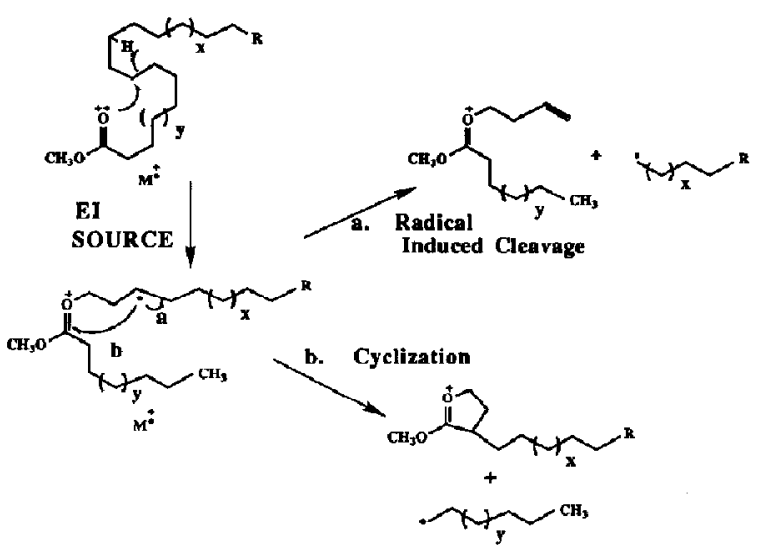

Scheme II

spectrum, would not form following collisional activation; however, decomposition could proceed by two competitive pathways to form the observed carbomethoxy ions: cyclization, with loss of the internal carbon skeleton (Scheme ITb) or radical-site induced scission of the alkyl chain and loss of the terminal carbon skeleton (Scheme Ila). This mechanism would not readily explain the extensive hydrogen-deuterium exchanges that compound the CID spectrum of methyl $\left[2,2-\mathrm{D}_{2}\right]$ octadecanoate (Figure 2); however, it is plausible that such hydrogen exchange reactions occurred in the source region during the formation of the alkylrearranged molecular ion.

Another striking feature of these low-energy CID mass spectra is the potential application to structure determination of saturated, branched fatty acid methyl esters. The regular pattern of the carbomethoxy ion series formed with lowenergy CID of $\mathrm{M}^{+-}$of saturated straight-chain fatty acid methyl esters was altered by methyl branches. The effect of the methyl substitution was to enhance the abundance of ions formed by $\alpha$-cleavage on either side of the branch carbon. This enhancement of ion formation was observed for both cyclization and radical cleavage, with exceptions determined by the minimum size of the alkyl radical loss or alkyl chain cyclized. As shown in Figure $4 \mathrm{~b}$, CID of methyl 13-methyl-pentadecanoate resulted in the enhanced abundance of $m / z 213,115$, and, to some extent, 241 . The ions at $m / z 213$ and 241 could arise from radical-induced cleavage of the carbon bonds between $C_{12}-C_{13}$ and $C_{13}-C_{14}$, respectively. Although the CID of the molecular ion of branched-chained hydrocarbons (formed by EI) was also found to generate abundant ions at each branch point [12], the mechanism of decomposition of these radical cations is likely to be very different.

This technique of low-energy CID of molecular ions generated by EI provides a sensitive, powerful, and simple approach to the determination of methyl branched, saturated fatty acids without the need for special derivatization $[12,13]$ or high-energy MS/MS $[13,14]$. The data presented were generated from less than 50 pmol of each methyl ester. This method combines the utility of gas chromatography, the facility of EI, and MS/MS for the analysis of isomeric fatty acids encountered in complex mixtures from biological extracts.

\section{Acknowledgments}

The authors thank Ms. Deborah Bcckworth for preparation of this manuscript. This work was supported in part by a grant from the National Institutes of Health (HL25785).

\section{References}

1. Ryhage, R.; Stenhagen, E. Arkw Kemi 1959a, 13, 523-533.

2. Ryhage, R.; Stentagen, E. Arkiv Kemi 1959b, 14, 483-495.

3. Ryhage, R.; Stenhagen, E. Arkiv Kemi 1959c, 14, 497-509.

4. Ryhage, R.; Stenhagen, E. Arkio Kenti 1959d, 15, 291-316.

5. McLafferty, F. W. Anal. Chem. 1959, 31, 82-87.

6. McCloskey, J. A. In Topics in Lipid Chemistry, Vol. 1; Gunstone, F. D., Ed.; Logos Press: London, 1970; pp. 369-440.

7. Dinh-Nguyen, N. Arkiv Kemi 1968, 28, 289-362.

8. Larsson, L.; Mardh, P.; Odham, G. J. Chromatogr. 1979, 163, 221-224.

9 Jensen, N. J., Gross, M. L. Lipids 1986, 21, 362-365.

10. Budzikiewicz, H.; Djerassi, C.; Williams, D. H. Mass Spectrometry of Organic Compounds; Holden-Day: San Francisco, 1967; pp. 3-17.

11. Adams, J.; Gross, M. L. Anal. Chem. 1987, 59, 1576-1582.

12. Cerny, R. L.; Pomonis, J. G; Fatland, C. F.; Nelson, D. R. Proc. Annu. Conf. Mass Spectrom. 1985, 33, 913-915.

13. Harvey, D. J., Biomed. Mass Spectrom. 1982, 9, 33-38.

14. Andersson, B. A. Prog. Chem. Fats Other Lipids 1978, 16, 279-308. 